\title{
Smart Stress Care: Usability, Feasibility and Preliminary Efficacy of Fully Automated Stress Management Application for Employees
}

\author{
Ji Hyun Baek¹, Jeong Hyun Kim² ${ }^{\circledR}$, Sohee $\mathrm{Oh}^{3}$, Ju Young Kim4, and Soyoung Baik ${ }^{2}$ \\ ${ }^{1}$ Department of Psychiatry, Samsung Medical Center, Seoul, Republic of Korea \\ ${ }^{2}$ Department of Psychiatry, Seoul National University Bundang Hospital, Seongnam, Republic of Korea \\ ${ }^{3}$ Department of Biostatistics, Seoul Metropolitan Government Seoul National University Boramae Medical Center, Seoul, Republic of Korea \\ ${ }^{4}$ Department of Family Medicine, Seoul National University Bundang Hospital, Seongnam, Republic of Korea
}

Objective We developed a fully automated smartphone-based stress management application and explored its usability, potential feasibility, and preliminary efficacy for stress management in Korean employees.

Methods Healthy employees working in large public enterprises were enrolled. Participants used our automated stress management application for four weeks. With the application, they monitored their stress level and life style factors. Personalized stress management techniques, including psychoeducation and cognitive behavioral technique, were also provided based on their stress level and lifestyle pattern. In 2014, additional relaxation techniques were incorporated. Participants' mental health status and lifestyle pattern were self-assessed at baseline and at 4 weeks after using the application.

Results A total of 68 subjects were recruited. The application generally received high satisfaction rating. After the intervention, perceived stress level was significantly decreased, both in 2013 and 2014 (BEPSI-K score pre. vs. post. 14.27 vs. $11.00, \mathrm{~F}=12.49$, $\mathrm{p}=0.001$ in 2013; 12.05 vs. $10.00, \mathrm{~F}=17.18, \mathrm{p}<0.001$ ). In 2014 , depression symptom severity was also significantly decreased (CES-D score pre- vs. Post-, 17.66 vs. $11.95, \mathrm{~F}=9.76, \mathrm{p}=0.004)$. The effects were more significant in females and in those $<35$ years.

Conclusion Our fully automated stress management application is acceptable and usable, showing preliminary efficacy for reducing employees' stress levels.

Psychiatry Investig 2018;15(10):991-999

Key Words Smartphone-based, Application, Stress management intervention, Employees.

\section{INTRODUCTION}

Stress of employees has been known as an important risk factor for major mental health problems. It is associated not only with the development of various mental health problems, but also with unhealthy lifestyle behaviors, eventually leading to negative consequences, including absenteeism, loss of productivity, and disability. ${ }^{1,2}$ For these reasons, early stress management has attracted much attention as the primary focus of the prevention of mental health problems. ${ }^{3}$

Although there various stress management interventions,

\footnotetext{
Received: March 23, 2018 Revised: July 3, 2018

Accepted: August 10, 2018

$\square$ Correspondence: Jeong Hyun Kim, MD, PhD

Department of Psychiatry, Seoul National University Bundang Hospital, 82 Gumi-ro 173beon-gil, Bundang-gu, Seongnam 13620, Republic of Korea

Tel: +82-31-787-2025, Fax: +82-31-787-4050, E-mail: retrial3@hanmail.net

(c) This is an Open Access article distributed under the terms of the Creative Commons Attribution Non-Commercial License (https://creativecommons.org/licenses/bync/4.0) which permits unrestricted non-commercial use, distribution, and reproduction in any medium, provided the original work is properly cited.
}

most of them have been underutilized, resulting in many affected employees left untreated. ${ }^{4}$ This may partly be due to limited accessibility, ${ }^{3,5}$ barriers in time or treatment cost, or even fear of potential stigmatization attached to mental health problems. These factors work as major barriers in implementing stress management interventions in Korea. ${ }^{6}$ According to a study conducted by the Korean society of occupational stress with approximately 7,000 workers from 254 businesses across the country, $673 \%$ of the subjects were exposed to potential occupational stress and $22 \%$ of the subjects were suffering from severe stress. However, psychiatric services were severely underutilized, likely due to fear of stigmatization and lack of knowledge about mental health services. ${ }^{7}$

Technological advances in the internet and smartphones may allow affected employees to have easier access to stress management interventions. In particular, a smartphone-based intervention may maximize its effects on reducing mental stress by overcoming barriers like time or place-providing flexibility to meet the individual's needs-as well as stigmatization 
by providing anonymity upon request. Considering cultural obstacles, i.e., social stigma, related to using mental health services and high penetration rate of smartphones ( $94 \%$ of all population $)^{8}$ in Korea, a smartphone-based stress management intervention can be a promising tool.

Hence, we developed a fully automated stress management application that is accessible 24 hours a day and can be utilized at the individual's own pace and convenience. In this study, we aimed to evaluate its usability, potential feasibility, and preliminary efficacy in stress management of employees.

\section{METHODS}

\section{Development process of the fully automated smartphone application}

We developed a smartphone application entitled "Stress Guide" for employees and a web-based system for mental health professionals in collaboration with KT, a Korean wireless communication company. KT assisted in the technical aspect of application development. The application was developed for iOS. Prior to development, we randomly distributed a questionnaire to employees in three public enterprises to explore what types of interventions were primarily desired by employees. The application was developed based on their feedback. The application was initially composed of four parts; 1) mental health and lifestyle assessment, 2) daily lifestyle management, and 3) forum for sharing their sources of trouble and getting psychological support among participants, including a frequently asked questions section, and 4) a tool for searching nearby mental health professionals.

After 4 weeks of intervention, we collected participants' feedback on their satisfaction using a 5-point Likert scale and asked open-ended question about additional features they wanted to see in the next iteration. After finishing the study in 2013, most participants wanted additional practical features that can help relaxation. Following their feedback, we also included 5) a relaxation training part in 2014.

\section{Recruitment}

Employees were recruited through our advertisement posted at Seoul National University Bundang Hospital (SNUBH) health promotion center (HPC), or by phone and/or e-mail correspondence to employees of three large public enterprises. SNUBH HPC provides standardized annual medical examinations, including lifestyle risk factor evaluations and laboratory tests, as well as national cancer screening services for employees. Any employees from one of these three enterprises or any employed individual who visited HPC for a health checkup were eligible to participate in this study. Another eligibility criterion for participation was smartphone literacy. After explaining the purpose and procedures, those who agreed to participate were included. Informed consent was received from all participants. This study was approved by the Institutional Review Board of SNUBH (B-1206/158-112).

\section{The contents of the app (Figure 1)}

\section{Mental health and lifestyle assessment part}

At baseline, participants evaluated their mental health status, occupational stress level, and lifestyle factors, including smoking, alcohol drinking, exercise, eating habits, and sleep. Once they filled out all the measures, test results were presented with a web diagram and detailed explanation about their mental health status. When clicking on each section of the diagram, results were automatically provided with personalized recommendations from mental health professionals based on participants' test results (Figure 2). Recommendations were mainly composed of psychoeducation and cognitive-behavioral techniques to modify their maladaptive thoughts and behaviors.

\section{Daily mood with life style monitoring}

Participants were also able to voluntarily monitor their daily lifestyle pattern, including includes alcohol consumption, smoking, coffee intake, sleep, exercise, and dietary patterns

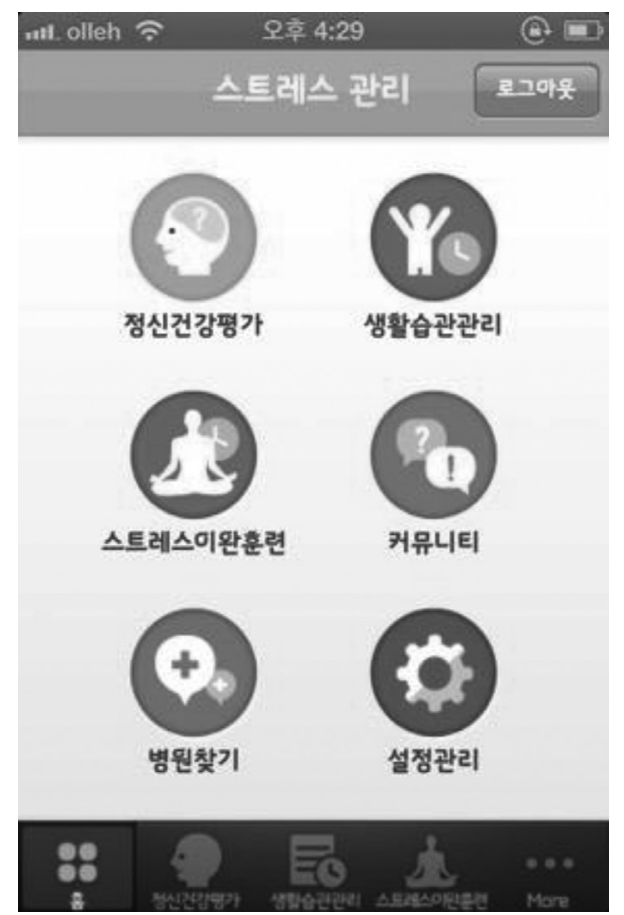

Figure 1. Main screen of the application. Top title is stress management. Six main icons are presented on the screen. Clockwise from the left top corner: mental health evaluation, life style management, Forum, settings, a tool for searching nearby mental health professionals, and relaxation technique training. 


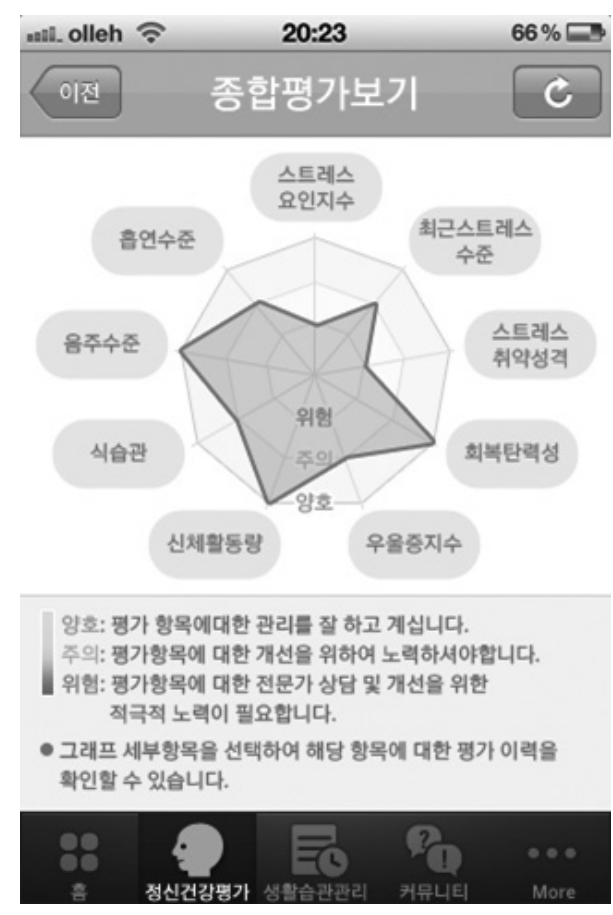

Figure 2. Results of the mental health and lifestyle assessment. At the top of the screen: Test results. When a participant finishes the baseline evaluation, including mental health evaluation and lifestyle assessment, the result was shown as a diagram that summarizes the results of all sections. On the diagram, clockwise from the top center: current stress factor score, recent perceived stress level, stress vulnerable personality (which indicated a Type A personality), resilience, depression, physical activity, eating habits, alcohol consumption, and smoking. If the score is higher than the cut-off score, the dot on each item is located close to the center. At the bottom: green means that the participant is managing the item well, yellow means that the participant needs improvement on the item, red means that the participant needs active involvement to improve this item. When clicking each section of the test on the diagram, results are provided with personalized recommendations from mental health professionals based on users' test results. Recommendations are mainly composed of psychoeducation and cognitive-behavioral techniques to modify their maladaptive thoughts and behaviors.

with simple mood evaluation on a daily, weekly, and monthly basis. When they answer 10 brief questions regarding lifestyle management on the app, they are provided with a short, customized comment with recommendations to improve daily habits, which is automatically generated based on the score from their daily condition. Participants can set push alarms to be notified at certain times of the day for self-evaluation.

\section{Forum and frequently asked question part}

In the forum section, participants were able to share their problems with other users anonymously. Additionally, participants had free access to the frequently asked questions (FAQs) section, which addressed topics relating to common stressors of employees, including marital problem, interpersonal relationship, career development, coworker relationship, child rearing, in-law issues, emotional problems, and general self-management. This section was answered by employee assistance program professionals with more than three years of experience in stress management programs.

\section{A tool for searching nearby mental health professionals}

In case participants wanted an offline mental health service, our application provided a search tool to help find nearby mental health professionals. The search results provided a list of professionals with their addresses, phone numbers, and direct links to their websites.

\section{Relaxation training part}

Following the participants' feedback in 2013, we additionally included the relaxation training part in 2014. All 38 participants who took part in this study during 2014 were able to use this feature. Abdominal breathing, progressive muscular relaxation, and meditation were included. A voice guide was provided to each relaxation method. Participants can choose the gender of voice guide and background music. Logs were left on the calendar for participants to monitor their application activity.

\section{Implementation}

After obtaining informed consents, an iPhone with the preinstalled stress guide application was provided to participants who did not already possess an iPhone during the 4 -week study period. For those who already had an iPhone, instruction on installing the application was provided. A baseline evaluation was self-assessed using measures included in the mental health and lifestyle assessment part of the application. Participants were able to use the fully automated application during the study period without limit at no cost. Prior to participation, participants were instructed to use the app at least twice or three times a week. No direct human contact was made to encourage or monitor their usage. Instead, a brief text message-once during the study period-was sent to encourage usage of the application. After four weeks, a final self-assessment was done, with the same measures used for the baseline evaluation, and the rented iPhones were collected at this time. All self-assessed data were automatically sent and stored in the internet system for analysis and were available for download as excel files.

After 4 weeks' intervention, we collected participants' feedback on satisfaction using a 5-point Likert scale and asked open-ended question regarding additional feature that users might want for the next iteration. Following the participants' feedback in 2013, we also included the relaxation training part in 2014. 


\section{Assessment measures}

Changes in depressive symptoms, perceived stress levels, and lifestyles were monitored at baseline evaluation and the final evaluation. Among them, changes in individual's perceived stress level and depressive symptoms were used as primary outcome measures. Changes in occupational stress level, resilience, and lifestyle were also additionally evaluated.

\section{Primary symptom outcomes}

Changes in individual's perceived stress level and depressive mood were used as the primary outcome measures. Individual's perceived stress level was evaluated using the Korean version of the brief encounter psychosocial instrument (BEPSI-K). ${ }^{9}$ The brief encounter psychosocial instrument (BEPSI) evaluates the subjectively perceived stress levels using five questions with a 5-point Likert scale and is a widely used measure of perceived stress with high reliability and validity. ${ }^{10}$ Likewise, the Korean version, BEPSI-K, also showed high reliability and validity. ${ }^{9}$

Changes in depressive mood were evaluated using the center for epidemiologic studies depression scale (CES-D). ${ }^{11}$ The CES-D is a 20 -item questionnaire with a 5-point Likert scale used for screening depression in the general population. The Korean translated version of CES-D also showed high reliability and validity. ${ }^{12}$

\section{Additional measures}

The social readjustment rating scale (SRRS) ${ }^{13}$ was applied to measure the current stress factors that can work as a confounding factor in evaluating psychological distress. The SRRS $^{13}$ assesses the experiences of 43 major stressful events during the past year. Each event has a different weight depending on its severity, and the total score generates the intensity of recent stressors, which can work as a confounding factor in evaluating psychological distress.

Type A personality-generally known to be vulnerable to stress-was evaluated using Framingham type A behavior pattern (FTA). ${ }^{14}$ The FTA contains a 20 -item questionnaire with an 11-point Likert scale.

The Korean occupational stress scale (KOSS) brief version ${ }^{15}$ was additionally used to evaluate stress levels related to occupational settings. KOSS uses 24 questions with a 4-point Likert scale. The KOSS brief version is composed of seven subscales used to evaluate the main causes of occupational stress, including job demand, insufficient job control, interpersonal conflict, job insecurity, occupational system, lack of reward, and organizational climate.

Individual's resilience was evaluated using the Korean resilience quotient-53 (KRQ-53). ${ }^{16}$ The KRQ-53 has 53 questions with a 5-point Likert scale, which evaluates various compo- nents of resilience, including controllability, positivity, and sociability.

In evaluating exercise and diet patterns, the international physical activity questionnaire (IPAQ) ${ }^{17}$ and the mini dietary assessment (MDA) ${ }^{18}$ were used. IPAQ has 6 questions regarding the duration and intensity of exercise in the past one week. A higher score indicates high activeness. The IPAQ scores were classified into three categories: 1) the 'active' category, which was characterized with intense exercise at least three times a week with a total IPAQ score of $\geq 1500$ or exercise every day with a total IPAQ score of $\geq 3000 ; 2$ ) the 'minimally active' defined by 20 minutes of intense exercise at least three times a week or 30 minutes of light exercise every day, or a total IPAQ score of $\geq 600$; and 3 ) the 'non-active' category, which includes other cases not previously defined. MDA evaluates whether the individual maintains a regular and balanced diet with 6 questions; a higher score means healthier diet.

Smoking and alcohol consumption patterns were explored using the Fagerström test for nicotine dependence (FTND) ${ }^{19}$ and the Korean version of the alcohol use disorders identification test (AUDIT), ${ }^{20}$ respectively. FTND contains six items regarding nicotine craving. The sum of the individual item scores is associated with biological nicotine dependence. ${ }^{19}$ AUDIT is a screening questionnaire that uses 10 sub-items to identify individuals with hazardous and harmful patterns of alcohol consumption. In both questionnaires, higher scores indicate greater dependence.

\section{Usability assessment}

At the final visit, we collected participants' feedback on satisfaction using a 5-point Likert scale and asked open-ended questions about additional features that they want included in the next iteration. Their satisfaction about each content, speed, and design of the application was measured with a 5-point Likert scale. We also asked their willingness to pay, and how much, for continual usage of the application.

\section{Statistical analysis}

Descriptive data for the sample were presented as the mean with standard deviation (SD) for continuous variables and counts with proportions for categorical variables. Symptom outcome measures were compared between the baseline and final evaluation using a generalized linear mixed effects model analysis, which is one of methods for analyzing repeated measures. It can build models for continuous or categorical outcomes while accounting for correlations between the outcomes from the same subject. To adjust the effects of recent stressful events an individual may have experienced, SRRS, recruitment year, gender, and age $[<35$ years or not (median)] were applied as covariates. These were chosen by Akaike in- 
formation criterion (AIC) $)^{21}$ and Bayesian information criterion (BIC) $)^{22}$ as measures of model (variable) selection. Moreover, in order to determine the effects of gender and age, the same analyses were conducted after dividing the groups by gender and age. Thirty-five years was the median age, which was also associated with the duration of employment. Twenty-seven (79.4\%) subjects aged $\geq 35$ years were employed for more than 10 years, while only $7(20.6 \%)$ subjects aged $<35$ years were employed for more than 10 years. Thus, we created subgroups depending on age; a group younger than 35 years and a group older than 35 years. To control for familywise error rates, Bonferroni correction was applied for multiple testing.

Statistical analysis was performed by using $r$ version 3.2.1 (http://www.r-project.org), and the level of significance was set at $\mathrm{p}<0.05$.

\section{RESULTS}

A total of 68 participants took part in this study (Table 1). All participants attended the final visit; no one dropped out. Based on self-reported questionnaire, the frequency of application usage was reported as 2.7 times per week and $23.9 \mathrm{~min}$ utes on average per use.

\section{Outcome measures (Table 2)}

After four weeks of intervention, the BEPSI-K score was significantly reduced in both 2013 and $2014(\mathrm{~F}=12.49, \mathrm{p}=0.001$ in 2013; $\mathrm{F}=17.18, \mathrm{p}<0.001$ in 2014). The CES-D score ( $\mathrm{F}=9.756$, $\mathrm{p}=0.004$ ) was additionally decreased in 2014. No significant differences were observed between the participants recruited in 2013 and those in 2014 with respect to changed patterns of measures.

We conducted a power analysis for the primary outcome

Table 1. Demographic data of participants

\begin{tabular}{lccc}
\hline & $\begin{array}{c}\text { Participants in } 2013 \\
(\mathrm{~N}=30)\end{array}$ & & $\begin{array}{c}\text { Participants in } 2014 \\
(\mathrm{~N}=38)\end{array}$ \\
\cline { 2 - 2 } Gender & $\mathrm{N}(\%)$ or mean $\pm \mathrm{SD}$ & & $\mathrm{N}(\%)$ or mean $\pm \mathrm{SD}$ \\
Male & $19(63.33)$ & & $9(23.68)$ \\
Female & $11(36.67)$ & & $29(76.32)$ \\
Age & $34.80 \pm 7.96$ & & $36.45 \pm 10.64$ \\
Duration of employment (year) & & \\
$<3$ & $12(40.00)$ & & $13(34.21)$ \\
$3-5$ & $2(6.67)$ & & $7(18.42)$ \\
$5-10$ & $6(20.00)$ & $4(10.53)$ \\
$\geq 10$ & $10(33.33)$ & $14(36.84)$ \\
\hline
\end{tabular}

SD: standard deviation

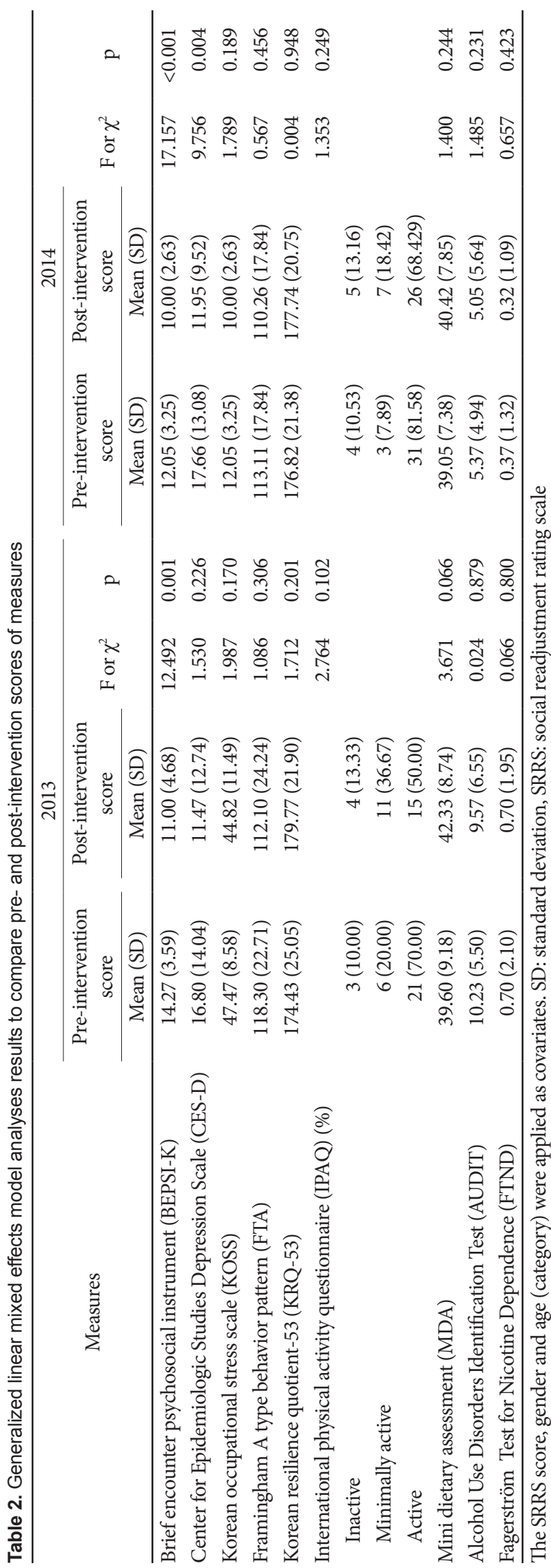


measures. The power of BEPSI-K in 2013 and 2014 was $94.23 \%$ and $98.54 \%$ at $\alpha=0.05$, respectively. Additionally, the CES-D showed a power of $88.77 \%$ at $\alpha=0.05$ after correcting for multiple testing; only the BEPSI-K in 2014 showed satisfactory power at $\alpha=(0.05 / 9)$.

We conducted additional analyses to explore the effect of age and gender (Table 3). Considering that age was strongly associated with duration of employment, we stratified participants by age ( $<35$ vs. $\geq 35$ years).

When participants were stratified by gender, males showed a significant decrease, only in the BEPSI-K score, while females showed a significant decrease in the BEPSI-K, FTA, CES-D, and AUDIT scores.

When participants were stratified by age, those $<35$ years showed a significant decrease in the scores of BEPSI-K, CES$\mathrm{D}$, and IPAQ, and an increase in the MDA score. Those $\geq 35$ years showed a significant decrease in the BEPSI-K and KOSS scores.

We conducted additional analyses to explore the effect of application usage duration (Table 4). The median duration of usage per week was 40 minutes, so we divided users into two group based on their usage duration using the median duration as cut-off point (average use frequency per week $\times$ average spent time per use). When participants were stratified by application usage duration, participants who used the app more than 40 minutes per week showed a significant improvement in the scores of BEPSI-K, CES-D, KOSS, KRQ-53, and AUDIT, while those who used the app less than 40 minutes showed a significant improvement only in the BEPSI-K and MDA scores.

\section{Usability of the application}

Participants generally reported high satisfaction with re-

Table 3. Generalized linear mixed effects model analyses by gender and age subgroup

\begin{tabular}{|c|c|c|c|c|c|c|c|c|}
\hline \multirow[t]{2}{*}{ Measures } & \multicolumn{2}{|c|}{$\begin{array}{c}\text { Males } \\
(\mathrm{N}=28)\end{array}$} & \multicolumn{2}{|c|}{$\begin{array}{l}\text { Females } \\
(\mathrm{N}=40)\end{array}$} & \multicolumn{2}{|c|}{$\begin{array}{c}\text { Participants } \\
<35 \text { yrs }(\mathrm{N}=34)\end{array}$} & \multicolumn{2}{|c|}{$\begin{array}{c}\text { Participants } \\
\geq 35 \text { yrs }(\mathrm{N}=34)\end{array}$} \\
\hline & $\mathrm{F}$ & $\mathrm{p}$ & $\mathrm{F}$ & $\mathrm{p}$ & $\mathrm{F}$ & $\mathrm{p}$ & $\mathrm{F}$ & $\mathrm{p}$ \\
\hline Brief encounter psychosocial instrument (BEPSI-K) & 10.737 & 0.003 & 16.189 & $<0.001$ & 18.481 & $<0.001$ & 10.084 & 0.003 \\
\hline Center for Epidemiologic Studies Depression Scale (CES-D) & 2.590 & 0.120 & 7.867 & 0.008 & 10.362 & 0.003 & 1.223 & 0.277 \\
\hline Korean occupational stress scale (KOSS) & 0.418 & 0.524 & 2.938 & 0.095 & 0.323 & 0.574 & 4.604 & 0.040 \\
\hline Framingham A type behavior pattern (FTA) & 0.004 & 0.952 & 5.049 & 0.031 & 3.257 & 0.081 & 0.267 & 0.609 \\
\hline Korean resilience quotient-53 (KRQ-53) & 0.135 & 0.716 & 1.235 & 0.273 & 3.390 & 0.075 & 0.009 & 0.925 \\
\hline International physical activity questionnaire (IPAQ) & 1.049 & 0.311 & 2.704 & 0.104 & 3.188 & 0.079 & 0.564 & 0.455 \\
\hline Mini dietary assessment (MDA) & 2.254 & 0.145 & 2.488 & 0.123 & 10.924 & 0.002 & 0.019 & 0.891 \\
\hline Alcohol Use Disorders Identification Test (AUDIT) & 0.095 & 0.760 & 9.592 & 0.004 & 2.189 & 0.149 & 0.000 & 0.986 \\
\hline
\end{tabular}

The SRRS score and recruited year were applied as covariates. SRRS: social readjustment rating scale

Table 4. Generalized linear mixed effects model analyses by application usage duration per week subgroup

\begin{tabular}{|c|c|c|c|c|}
\hline \multirow[t]{2}{*}{ Measures } & \multicolumn{2}{|c|}{$\begin{array}{c}\text { The average application usage duration } \\
\text { per week }<40 \text { minutes }(\mathrm{N}=36)\end{array}$} & \multicolumn{2}{|c|}{$\begin{array}{l}\text { The average application usage duration } \\
\text { per week } \geq 40 \text { minutes }(\mathrm{N}=32)\end{array}$} \\
\hline & $\mathrm{F}$ & $\mathrm{p}$ & $\mathrm{F}$ & $\mathrm{p}$ \\
\hline $\begin{array}{l}\text { Brief encounter psychosocial instrument } \\
\text { (BEPSI-K) }\end{array}$ & 18.185 & $<0.001$ & 11.189 & 0.002 \\
\hline $\begin{array}{l}\text { Center for Epidemiologic Studies Depression Scale } \\
\text { (CES-D) }\end{array}$ & 3.835 & 0.058 & 6.835 & 0.014 \\
\hline Korean occupational stress scale (KOSS) & 0.004 & 0.949 & 7.082 & 0.012 \\
\hline Framingham A type behavior pattern (FTA) & 0.099 & 0.755 & 3.341 & 0.078 \\
\hline Korean resilience quotient -53 (KRQ-53) & 0.582 & 0.451 & 5.714 & 0.023 \\
\hline International physical activity questionnaire (IPAQ) & 0.785 & 0.379 & 2.832 & 0.098 \\
\hline Mini dietary assessment (MDA) & 5.349 & 0.027 & 0.876 & 0.357 \\
\hline Alcohol Use Disorders Identification Test (AUDIT) & 0.118 & 0.733 & 4.255 & 0.048 \\
\hline Fagerström Test for Nicotine Dependence (FTND) & 1.086 & 0.305 & 3.008 & 0.093 \\
\hline
\end{tabular}

The SRRS score and recruited year were applied as covariates. SRRS: social readjustment rating scale 
spect to the overall usability of the application (4.0 points on a 5-point Likert scale). They were mostly content with appreciation meditation, ease of use, and speed of the app (4.26 points); 41 participants (60.3\%) replied that they were willing to pay money to use the application.

\section{DISCUSSION}

Our study suggests that our application may be an acceptable strategy to manage stress for employees in Korea. After a four-week intervention, perceived stress level and depressive symptoms were significantly decreased. No one dropped out before the end of the study, and they used the application 2.7 times a week on average. Participants were generally satisfied with their experiences.

It is notable that the CES-D score in our study was higher than the general population. ${ }^{23}$ Previous study with Korean employees also reported high CES-D scores (mean $=13.02$, $\mathrm{SD}=7.75$ for females; mean $=12.25, \mathrm{SD}=8.06$ for males). ${ }^{6} \mathrm{Ko}-$ rean employees generally suffer from high stress level and low job satisfaction due primarily to long working hours and strict occupational climate. ${ }^{24}$ It is also possible that employees who experienced psychological symptoms were more willing to participate in this study. ${ }^{25}$

Smartphone-based stress management intervention is a promising tool for managing mental health problems with high accessibility and availability. ${ }^{26-29}$ Intervention is made available to users anytime and anywhere, at their own leisure, with anonymity. Recently published studies showed that smartphone-based stress management intervention was effective in reducing mental health-related symptoms; $;^{27,28}$ however, evidences for its usability and acceptability are still scarce.

Smartphone-based interventions are beginning to emerge as a method of treatment for various mental health problems. However, it is important to carefully consider the characteristics of the equipment used when planning for content. ${ }^{30}$ Unlike face-to-face intervention or web-based intervention using desk-top computers, smartphone users can access interventions on their devices in any location for a relatively short duration..$^{30}$ Thus, content provided should accommodate this usage pattern. In our application, we tried to accommodate this characteristic by offering various types of interventions on-demand with time constraint in mind-each type requires only a few minutes to complete. Considering that traditional psychiatric interventions for clinical conditions (i.e., depression or anxiety disorder) require a longer time commitment with high intensity, smartphone-based intervention may not be a suitable replacement for the traditional intervention for clinical conditions. Smartphone-based stress management intervention targets relatively milder conditions with more resilient populations.

In this study, we did not provide direct human support. It is unclear whether this lack of direct human contact-unguided intervention-is effective in stress management. Several studies $^{31,32}$ reported unguided electronic (i.e., Desktop computer-based) mental health interventions were less effective than guided interventions, but they targeted clinical populations, who generally require more intensive treatment than employees with stress. Christenson et al. ${ }^{33}$ reported that depression severity significantly affected adherence to electronic intervention programs, suggesting that less severe mental health conditions may not require direct human support. Moreover, unlike desktop computers, smartphones can utilize push notifications, i.e., alarms, to remind users, as incorporated in our study.

A meta-analysis ${ }^{3}$ on the effects of stress management intervention showed that cognitive behavioral techniques generated the largest effects. However, it is unclear which method was most effective in the smartphone setting. In our study, we applied various techniques, including self-monitoring, psychoeducation, cognitive behavioral technique, and relaxation components, together and let users choose their favorite methods. Hence, it is unclear which technique was actually most effective for managing stress.

It should be noted that certain groups may respond better to smartphone-based intervention. This should be considered in the planning phases. In our study, we found that younger age ( $<35$ years of age) and female groups showed a greater significant decrease in depressive symptoms. Males and females generally showed different coping strategies and attitudes toward health problems. Previous studies showed that females put higher effort to overcome their mental health problems. ${ }^{34}$ This may contribute to females showing greater compliance with the self-guided intervention. ${ }^{33}$ In our study, females not only showed greater improvement in depressive symptoms and perceived stress levels, but they also showed improvement in type A personality patterns and alcohol use problems. This suggests that smartphone-based interventions can effectively prevent mental health problems in female employees.

The younger age group may be considered as target patient group for the smartphone-based intervention. However, in our study, those $\geq 35$ years showed significant improvement in KOSS scores while those $<35$ years did not. Previous studies also showed mixed results about the effect of age on adherence to electronic interventions. ${ }^{25,33}$ Further study is needed to better determine the role of age on the effects of smartphone-based intervention.

Smartphone-based application for mental health has become dramatically common recently. Following this trend, evidence-based recommendation for application develop- 
ment has also been made by experts. ${ }^{35}$ We retrospectively evaluated our application following their recommendation ${ }^{35}$ on cognitive behavioral therapy-based technique, which targets non-clinical populations with anxiety and low mood; selfmonitoring components, including log of past application use and push alarm for reminders; activity suggestions and mental health information; links to crisis support services; and simple and intuitive interface. However, our application lacked a component of gamification (i.e., encouraging people to use the app via rewards and internal triggers).

The study results need to be interpreted within the scope of this study design. First, we did not include wait-list control groups. Several previous studies with respect to electronic interventions have shown that wait-list control groups also improved without intervention. ${ }^{36}$ However, it is important to point out that in most of these studies, wait-list control groups also received a follow-up evaluation, which may work as symptom monitoring that can decrease maladaptive behaviors by increasing awareness of their behavioral patterns. Second, we did not use an objective measure (e.g., physiological changes) or the smartphone generated objective data (e.g., voice features and data on activity and phone usage) in evaluating stress levels. However, most previous studies used subjective measures only for monitoring psychological distress levels. Additionally, the true gold standard for measuring "perceived stress level" is subjective considering that stress can be only measured through one's own perception. Moreover, we did not have objective data generated within the system that kept track of the application usage, i.e., how often and how long the application was used. This type of information can provide valuable, accurate information on application usage. ${ }^{36}$ But instead, such information was based on self-reported data. Third, the functionality of application was a bit different between 2013 and 2014; we added a relaxation technique part according to the participants' need. This might cause some confusion in interpreting the results. Fourth, our evaluation period was relatively short (four weeks), limiting our ability to determine the long term effects of smartphone-based stress management intervention. However, this study provides preliminary evidence that smartphone-based stress management intervention has the potential to manage stress. Fifth, participants chose to participate in this study, suggesting that they could be more motivated to use the intervention more efficiently than the general population.

Despite several limitations, this study suggested potential possibilities of effective intervention for stress management through fully automated smartphone-based application. Our application allowed employees to have more accessibility to stress management intervention and showed its potential effectiveness on reducing depressive symptoms and stress lev- els. Additional studies are needed to further investigate feasibility, acceptability, and long-term effects of our application on preventing and managing mental health problems.

\section{Acknowledgments}

This work was supported by KT-SNUBH collaborative research fund.

Part of the data in this study was presented and selected as an outstanding poster at the conference of the Korean psychosomatic society on June, 2013.

\section{REFERENCES}

1. Lerner D, Henke RM. What does research tell us about depression, job performance, and work productivity? J Occup Environ Med 2008;50: 401-410.

2. Henderson M, Harvey SB, Overland S, Mykletun A, Hotopf M. Work and common psychiatric disorders. J R Soc Med 2011;104:198-207.

3. Richardson KM, Rothstein HR. Effects of occupational stress management intervention programs: a meta-analysis. J Occup Health Psychol 2008;13:69-93.

4. Stress in America ${ }^{\mathrm{TM}}$ : Missing the Health Care connection. American Psychological Association, Washington, DC. Available at: https://www. apa.org/news/press/releases/stress/2012/full-report.pdf. Accessed February $26,2016$.

5. 2013/2014 Staying@Work U.S. Executive Summary Report. Willis Towers Watson. Available at: https://www.towerswatson.com/en-US/Insights/IC-Types/Survey-Research-Results/2013/09/2013-2014-stayingatwork-us-executive-summary-report. Accessed February 28, 2016.

6. Park SG, Min KB, Chang SJ, Kim HC, Min JY. Job stress and depressive symptoms among Korean employees: the effects of culture on work. Int Arch Occup Environ Health 2009;82:397-405.

7. Kim JL, Cho J, Park S, Park EC. Depression symptom and professional mental health service use. BMC Psychiatry 2015;15:261.

8. The Demographic Digital Divde is Real and Pervasive. Pew Research Center. Washington DC. Available at: http://www.pewglobal.org/2016/02/22/ internet-access-growing-worldwide-but-remains-higher-in-advancedeconomies/technology-report-02-04/. Accessed February 29, 2016.

9. Yim J, Bae J, Choi S, Kim S, Hwang H, Huh B. The validity of modified Korean-translated BEPSI (Brief Encounter Psychosocial Instrument) as instrument of stress measurement in outpatient clinic. J Korean Acad Fam Med 1996;17:42-53.

10. Frank SH, Zyzanski SJ. Stress in the clinical setting: the Brief Encounter Psychosocial Instrument. J Fam Pract 1988;26:533-539.

11. Radloff LS. The CES-D Scale: a self report depression scale for research in the general population. Appl Psychol Meas 1977;1:385-401.

12. Cho MJ, Kim KH. Use of the Center for Epidemiologic Studies Depression (CES-D) Scale in Korea. J Nerv Ment Dis 1998;186:304-310.

13. Holmes T, Rahe R. The social readjustment rating scale. J Psychosom Res 1967;11:213-218.

14. Sohn Y, Yang Y, Kwon H, Lee Y, Lee J, Lee E. The association between type A behavior pattern and hypertension among adults in a rural area. Korean J Pub Health 2001;38:72-83.

15. Chang S, Koh S, Kang D, Kim S, Kang M, Lee C, et al. Developing an occupational stress scale for Korean employees. Korean J Occup Environ Med. 2005;17:297-317.

16. Shin W, Kim M, Kim J. Developing measures of resilience for Korean adolescents and testing cross, convergent, and discriminant validity. Stud Korean Youth 2009;20:105-131.

17. Sjostrom M, Ekelund U, Poortvliet E, Hurtig-Wennlof A, Yngve A. Assessment of physical activity using IPAQ (version 4) and activity monitors (CSA). Meas Phys Educ Exerc Sci 2000;4:263-264.

18. Kim W, Cho M, Lee H. Development and validation of mini dietary assessment index for Koreans. Korean J Nutr 2003;36:83-92.

19. Muhammad-Kah RS, Hayden AD, Liang Q, Frost-Pineda K, Sarkar M. The relationship between nicotine dependence scores and biomarkers 
of exposure in adult cigarette smokers. Regul Toxicol Pharmacol 2011;60:79-83.

20. Kim SS, Gulick EE, Nam KA, Kim SH. Psychometric properties of the alcohol use disorders identification test: a Korean version. Arch Psychiatr Nurs 2008;22:190-199.

21. Akaike H. A new look at the statistical model identification. IEEE Trans Autom Control 1974;19:716-723.

22. Schwartz G. Estimating the dimension of a model. Ann Stat 1978;6:461464.

23. Oh DH, Kim SA, Lee HY, Seo JY, Choi BY, Nam JH. Prevalence and correlates of depressive symptoms in korean adults: results of a 2009 korean community health survey. J Korean Med Sci 2013;28:128-135.

24. SERI. Worker happiness in Korea. Korea Economic Trends Weekly Insight 2013;775.

25. Crisp DA, Griffiths KM. Participating in online mental health interventions: who is most likely to sign up and why? Depress Res Treat 2014; 2014:790457.

26. Ahtinen A, Mattila E, Valkkynen P, Kaipainen K, Vanhala T, Ermes M, et al. Mobile mental wellness training for stress management: feasibility and design implications based on a one-month field study. JMIR Mhealth Uhealth 2013;1:e11.

27. Ebert DD, Heber E, Berking M, Riper H, Cuijpers P, Funk B, et al. Selfguided internet-based and mobile-based stress management for employees: results of a randomised controlled trial. Occup Environ Med 2016; $73: 315-323$.

28. Heber E, Lehr D, Ebert DD, Berking M, Riper H. Web-based and mo- bile stress management intervention for employees: a randomized controlled trial. J Med Internet Res 2016;18:e21.

29. Birney AJ, Gunn R, Russell JK, Ary DV. MoodHacker mobile web app with email for adults to self-manage mild-to-moderate depression: randomized controlled trial. JMIR Mhealth Uhealth 2016;4:e8.

30. Ben-Zeev D, Schueller SM, Begale M, Duffecy J, Kane JM, Mohr DC. Strategies for mHealth research: lessons from 3 mobile intervention studies. Adm Policy Ment Health 2015;42:157-167.

31. Richards D, Richardson T. Computer-based psychological treatments for depression: a systematic review and meta-analysis. Clin Psychol Rev 2012;32:329-342.

32. Johansson R, Andersson G. Internet-based psychological treatments for depression. Expert Rev Neurother 2012;12:861-869; quiz 870.

33. Christensen H, Griffiths KM, Farrer L. Adherence in internet interventions for anxiety and depression. J Med Internet Res 2009;11:e13.

34. Babwah F, Baksh S, Blake L, Cupid-Thuesday J, Hosein I, Sookhai A, et al. The role of gender in compliance and attendance at an outpatient clinic for type 2 diabetes mellitus in Trinidad. Rev Panam Salud Publica 2006;19:79-84.

35. Bakker D, Kazantzis N, Rickwood D, Rickard N. Mental Health Smartphone Apps: Review and Evidence-Based Recommendations for Future Developments. JMIR Ment Health 2016;3:e7.

36. Proctor EK, Powell BJ, McMillen JC. Implementation strategies: recommendations for specifying and reporting. Implement Sci 2013;8: 139. 